\title{
Processing the presence, placement, and properties of a distractor in spatial language tasks
}

\author{
laura A. Carlson and Patrick L. Hill \\ University of Notre Dame, Notre Dame, Indiana
}

\begin{abstract}
A common way to describe the location of an object is to spatially relate it to a nearby object. For such descriptions, the object being described is referred to as the located object; the object to which it is spatially related is referred to as the reference object. Typically, however, there are many nearby objects (distractors), resulting in the need for selection. We report three experiments that examine the extent to which a distractor in the display is processed during the selection of a reference object. Using acceptability ratings and production measures, we show that the presence and the placement of a distractor have a significant impact on the assessment of the spatial relation between the located and reference objects; there is also evidence that the properties of the distractor are processed, but only under limited conditions. One implication is that the dimension that is most relevant to reference object selection is its spatial relation to the located object, rather than its salience with respect to other objects in the display.
\end{abstract}

We interact with objects in a variety of ways: We recognize them, we act on them, and we speak about them. Each of these processes requires selecting an intended object from a set of nonintended objects. For example, with respect to recognition, if I want to make a phone call, I need to distinguish my cell phone from the objects surrounding it on my desk. With respect to language, I can convey the location of the cell phone to a listener who may want to use it. For example, I could spatially relate it to another object in the scene, as in The cell phone is behind the book. In this description, the cell phone is the located object, and the book is the reference object. Selection in this case includes not only the located object, but also the reference object, given the many objects surrounding the cell phone. Indeed, one could describe the cell phone's location in different ways, depending on the reference object selected, such as to the left of the monitor, in front of the coffee cup, or at the corner of the desk. The goal of the present article is to more closely investigate conditions in which a reference object must be selected in the presence of an additional distractor. We are specifically interested in the degree to which the presence, placement, and properties of multiple objects are evaluated during reference object selection.

\section{The Role of Salience in \\ Reference Object Selection}

It is typically assumed that a reference object is selected on the basis of properties that make it salient relative to other surrounding objects, and therefore easy to find (de Vega, Rodrigo, Ato, Dehn, \& Barquero, 2002; Miller \& Johnson-Laird, 1976; Talmy, 1983). Such an assump- tion is consistent with work in communication that argues that referential descriptions that include distinct attributes that make an object stand out in contrast to surrounding objects are particularly helpful in disambiguating the intended referent (Brown-Schmidt, Byron, \& Tanenhaus, 2005; Eberhard, Spivey-Knowlton, Sedivy, \& Tanenhaus, 1995; Olson, 1970). For example, Olson argued that a speaker would describe the same small round white block as the white one when in the context of a small round black block, and as the round one when in the context of a small square white block.

There are many possible properties that could potentially define salience, including perceptual, conceptual, and spatial features. With respect to perceptual features, Talmy (1983) argued that the spatial description The bicycle is near the house is more acceptable than The house is near the bicycle because the house is larger and more permanently situated, rendering it a more stable and hence more preferred reference object. In addition to size and stability, Talmy argued that reference objects may have greater geometric complexity. In addition, using a corpora of spatial descriptions, de Vega et al. (2002) found that reference objects were more likely to be (1) inanimate, (2) solid in their consistency, and (3) whole entities or mass objects (e.g., a beach or an ocean) rather than parts of an object. Blocher and Stopp (1998) defined salience with respect to color, shape, and size. Finally, for spatial sentences including multiple reference objects, Plumert, Carswell, De Vet, and Ihrig (1995) showed that the order of mention was hierarchical, either moving from the largest units to the smallest when giving directions (e.g., look in the basement in the laundry room on the washing ma- 
chine in a measuring cup) or in the reverse order from smallest to largest when providing descriptions.

With respect to conceptual features, Talmy (1983) suggested that a reference object would be less recently introduced to a scene than the other objects. Another type of conceptual feature is the functional relationship between the located and reference objects. For example, CarlsonRadvansky and Tang (2000) found that participants rated sentences as more acceptable descriptions of scenes containing two objects when the objects performed a typical interaction (i.e., a mustard bottle above a hamburger) than when they did not (a pesticide bottle above a hamburger). (For reviews of similar object effects on spatial language, see Carlson \& van der Zee, 2005; Coventry \& Garrod, 2004.)

With respect to spatial features, Craton, Elicker, Plumert, and Pick (1990) showed that children prefer to choose reference objects that stand in a front/back rather than left/right relation to the located object. This pattern is consistent with a larger literature showing preferences for the above/below vertical axis and front/back horizontal axis over the horizontal left/right axis, on the basis of correspondences with gravity (for abovelbelow) and our typical regions of movement and perception (for front/back) that help to distinguish these axes (Clark, 1973; Fillmore, 1971). In addition to these across-term preferences, there are also within-term preferences, such that some configurations are deemed better examples of a given spatial term than others (Hayward \& Tarr, 1995; Logan \& Sadler, 1996); for example, the description $X$ is above $O$ is preferred for a scene containing an "X" directly above an "O" over a scene containing an " $X$ " diagonally above and to the left of "O." Relative distance also influences reference object selection, with closer objects being preferred (Hund \& Plumert, 2007). Finally, for descriptions containing multiple landmarks, Plumert, Ewert, and Spear (1995; see also Plumert, Carswell, et al., 1995 ) showed that 3-and 4-year-olds found it easier to provide descriptions that included support relations (e.g., It's in the basket on the table) over proximal relations (e.g., It's in the basket next to the table).

\section{Selecting a Reference Object in the Presence of a Distractor}

The research described above identified various perceptual, conceptual, and spatial features that may all potentially combine to make an object salient, with such salience presumably rendering the object more likely to be selected as a reference object. This work all presumes that attributes of all of the objects in a given scene are compared and contrasted on (at least some of) these dimensions prior to selection. However, the evidence that the features of the other candidate objects (e.g., distractors) are processed is largely indirect, based on inferences drawn from the reference object that was ultimately selected. In the present article, we systematically manipulated the presence, placement, and properties (perceptual, conceptual, spatial) of a distractor in a more direct test of whether these attributes are processed.

In a prior study, Carlson and Logan (2001) tested whether the spatial relation between a distractor and the reference object was computed. Across trials, they ma- nipulated whether a distractor was present in displays containing located and reference objects. When the distractor was present, its placement relative to the other objects was manipulated. Figure 1 shows three possible locations of the distractor (differentiated as $\mathrm{D}_{1}, \mathrm{D}_{2}$, and $\mathrm{D}_{3}$ ), although only a single distractor was shown on any given trial. The task was to determine whether a description such as $L$ is above $R$ was acceptable for a given display. Spatial terms such as above refer to space surrounding the reference object that is delineated into regions (Hayward \& Tarr, 1995; Logan \& Sadler, 1996). In Figure $1, D_{1}$ is in the good region, directly above $\mathrm{R} ; \mathrm{D}_{2}$ is in the acceptable region, above and off to the side of $\mathrm{R}$; and $\mathrm{D}_{3}$ is in the bad region, not in an above relation at all. Given the placement of $\mathrm{L}$ in an acceptable region in Figure $1, \mathrm{D}_{1}$ is in a region that better exemplifies the above relation; $\mathrm{D}_{2}$ is in the same region, and is thus an equivalent example of the above relation; and $\mathrm{D}_{3}$ does not exemplify above at all. If the relation between the distractor and the reference object is computed, then one might expect judgments of the relation between the located object and reference object to differ as a function of whether the relation between the distractor and reference object is a better, equivalent, or poorer example of above. However, Carlson and Logan found that performance was not influenced by the placement of the distractor across these regions. On the basis of these results, and consistent with Logan and Compton (1996), Carlson and Logan argued that whereas the distractor had an impact on finding the relevant objects in the display, there was no evidence that its placement was processed (see also Greenspan \& Segal, 1984).

\section{Overview of the Experiments}

The present study sought evidence for the processing of the presence, placement, and properties of the distractor with different stimuli and across different tasks. With respect to stimuli, the displays used by Carlson and Logan (2001; see also Logan \& Compton, 1996) were made up

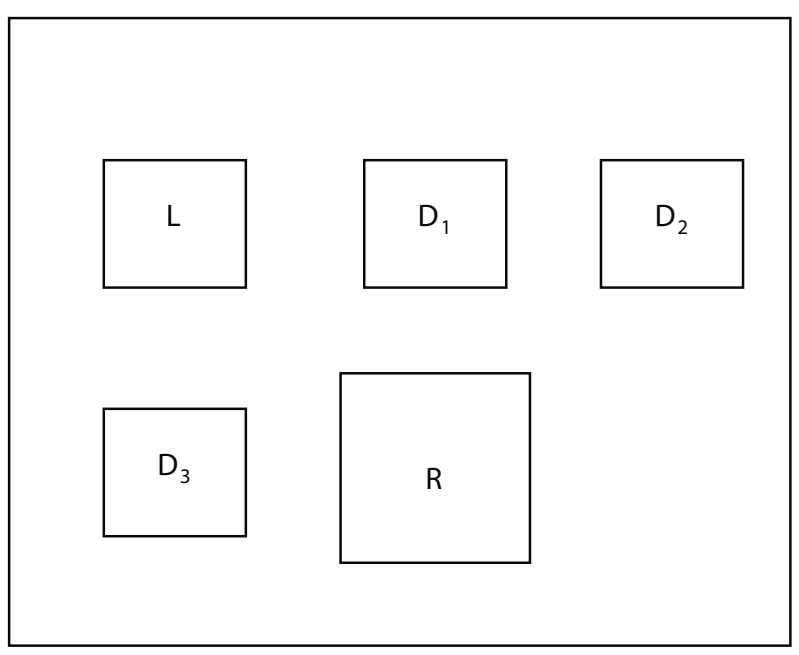

Figure 1. Illustration of placements of a located object $(L)$, reference object $(R)$, and three possible distractors $\left(D_{1}, D_{2}\right.$, and $\left.D_{3}\right)$. On any given trial, only one of the distractors was presented. 
of random consonant letters that do not typically interact. Indeed, vowels were excluded, to avoid the incidental presentation of words or pseudowords. However, considerable research has shown that object properties and the depicted interaction between the objects have a significant impact on the judgments of spatial relations (for a review, see Coventry \& Garrod, 2004). For example, with respect to projective spatial relations such as above, Carlson-Radvansky and Tang (2000) showed that ratings of acceptability were significantly affected by whether the objects were depicted in a manner consistent with the way in which they interact, suggesting that participants were not only assessing the spatial relation between isolated objects, but also assessing their functional properties. These effects have also been observed when participants were asked to place a located object above/below a reference object; such placements were significantly affected by the functional interaction between the objects (CarlsonRadvansky, Covey, \& Lattanzi, 1999). Similarly, Carlson and Kenny (2006) have reported data that suggest that participants simulate interactions between objects while interpreting their spatial relation. Given these stimulus effects, Experiment 1 sought evidence for processing of the distractor using objects that interact. The critical question was whether effects due not only to the presence of the distractor, but also to its placement and its properties, would now be observed.

With respect to task, Herskovits (1986) has claimed that on communicative grounds one would not use $L$ above $R$ to describe the relation between " $L$ " and " $R$ " in Figure 1 in the presence of $\mathrm{D}_{1}$, because $\mathrm{D}_{1}$ stands in better above relation with respect to "R." This claim is from the perspective of a speaker; however, the tasks employed previously (see Carlson \& Logan, 2001; Logan \& Compton, 1996) were from the perspective of a comprehender who was provided with sentences that specified the located object and reference object, and who simply determined the acceptability of these sentences as descriptions. Thus, the goal of Experiment 2 was to assess the generality across tasks of the effects observed in Experiment 1, while also more directly testing Herskovits's claim from the perspective of a speaker. Specifically, participants were shown the displays of objects from Experiment 1 and were asked to describe the location of a given object. Note that this is equivalent to the example in the introduction in which there are multiple objects that can serve as reference objects, resulting in the need for selection. In the experimental displays, one of the objects was presumed to be the preferred reference object on the basis of its salience on perceptual and conceptual grounds and physical properties. The critical question was whether there would be systematic changes in the selection of this salient object as the reference object as a function of the placement and properties of the other, less salient candidate reference object.

Finally, across all experiments, we manipulated the functional properties of the distractor and the located and reference objects, and the manner in which they were depicted. In Carlson-Radvansky and Tang (2000), the scenes contained objects that interacted either typically (such as a bottle of mustard and a hamburger) or atypically (such as a bottle of pesticide and a hamburger). Across scenes, the orientation of the objects was manipulated, so that sometimes the bottle was pointing downward in a position to enable one to successfully squirt the contents onto the hamburger, and sometimes the bottle was pointing upward, with the nozzle away from the hamburger. Importantly, the spatial configuration between the objects remained the same. There were two critical findings. First, in some conditions, ratings for the same spatial relation were lower when the objects interacting were atypical (e.g., pesticide and hamburger) than when they were typical (e.g., mustard and hamburger). Second, and more strongly, ratings for the same spatial relation were lower if the objects were depicted as not interacting (e.g., mustard pointing away from hamburger) than when they were depicted as interacting (e.g., mustard pointing toward hamburger).

To assess whether the properties of the distractor were processed during reference object selection, in Experiments 1 and 2, the objects were depicted as interacting, and the typicality of the interaction was varied. Experiment 3 manipulated both whether the objects were depicted as interacting and the typicality of the objects. Across the experiments, systematic effects due to typicality and to the depicted interactions among the objects would be indicative of the processing of the functional properties of the distractor in addition to its presence and placement.

\section{EXPERIMENT 1}

The goal of Experiment 1 was to assess whether the presence, placement, and properties of a distractor would be processed when using photos of real objects that were presented in an interactive manner. Following Carlson and Logan (2001), we used an untimed acceptability rating task. We also used the display configurations from Carlson and Logan (2001); these are shown in Figure 2. The two major columns correspond to the placement of the located object in either a good region directly above the reference object (designated LG) or in an acceptable region above but to the side of the reference object (designated LA). Within each major column, condition labels and sample displays are provided; for the displays, the positions of the boxed letters indicate their placement in the configuration (L, located object; R, reference object; D, distractor). The rows of the table correspond to the various placements of the distractor. Specifically, row 1 shows the configurations with the distractor absent; row 2 shows configurations with the distractor placed in a good region; row 3 shows configurations with the distractor in an acceptable region; and rows 4 and 5 show configurations with the distractor placed in the bad region. ${ }^{1}$

If the presence of the distractor is processed, then ratings for distractor-absent configurations should be higher, reflecting greater acceptability, than ratings for distractorpresent configurations, independent of distractor placement. This would replicate the findings observed by Logan and Compton (1996) and Carlson and Logan (2001). If the placement of the distractor is processed, then effects within the distractor-present configurations should be observed. Specifically, holding constant the placement 


\begin{tabular}{|c|c|c|c|c|c|c|}
\hline & \multicolumn{3}{|c|}{$\begin{array}{l}\text { Located Object in } \\
\text { Good Region }\end{array}$} & \multicolumn{3}{|c|}{$\begin{array}{l}\text { Located Object in } \\
\text { Acceptable Region }\end{array}$} \\
\hline & \multicolumn{2}{|c|}{$\begin{array}{l}\text { Display \& } \\
\text { Condition }\end{array}$} & Data & \multicolumn{2}{|c|}{$\begin{array}{l}\text { Display \& } \\
\text { Condition }\end{array}$} & Data \\
\hline $\begin{array}{l}\text { Distractor } \\
\text { absent }\end{array}$ & LG & $\mathrm{L}$ & $\begin{array}{l}\text { Rating } \\
\text { Typ: } 8.8(.10) \\
\text { Atyp: } 8.8(.10) \\
\text { \% Selected } \\
\text { Typ: } 100(0) \\
\text { Atyp: } 99(.5)\end{array}$ & $\begin{array}{l}\text { LA } \\
\qquad \mathrm{L}\end{array}$ & & $\begin{array}{l}\text { Rating } \\
\text { Typ: } 6.0(.24) \\
\text { Atyp: } 5.9(.25) \\
\text { \% Selected } \\
\text { Typ: } 98(2) \\
\text { Atyp: } 99(1)\end{array}$ \\
\hline $\begin{array}{l}\text { Distractor in good } \\
\text { region }\end{array}$ & & & & $\begin{array}{r}\text { LA/DG } \\
\mathrm{L}\end{array}$ & $\mathrm{R}$ & $\begin{array}{l}\text { Rating } \\
\text { Typ: } 4.2(.34) \\
\text { Atyp: } 4.2(.36) \\
\text { \% Selected } \\
\text { Typ: } 25 \text { (6) } \\
\text { Atyp: } 24(7)\end{array}$ \\
\hline $\begin{array}{l}\text { Distractor in } \\
\text { acceptable region }\end{array}$ & $\begin{array}{r}\mathrm{LG} / \mathrm{DA} \\
\mathrm{D}\end{array}$ & $\mathrm{R}$ & $\begin{array}{l}\text { Rating } \\
\text { Typ: } 8.3(.20) \\
\text { Atyp: } 8.1(.22) \\
\text { \% Selected } \\
\text { Typ: } 85 \text { (5) } \\
\text { Atyp: } 84 \text { (5) }\end{array}$ & $\begin{array}{l}\text { LA/DA } \\
\mathrm{L}\end{array}$ & D & $\begin{array}{l}\text { Rating } \\
\text { Typ: } 5.1(.31) \\
\text { Atyp: } 5.1(.33) \\
\text { \% Selected } \\
\text { Typ: } 51 \text { (7) } \\
\text { Atyp: } 51 \text { (8) }\end{array}$ \\
\hline $\begin{array}{l}\text { Distractor in bad } \\
\text { region }\end{array}$ & $\begin{array}{l}\text { LG/DB } \\
\mathrm{D}\end{array}$ & $\frac{\mathrm{L}}{\mathrm{R}}$ & $\begin{array}{l}\text { Rating } \\
\text { Typ: } 8.5(.17) \\
\text { Atyp: } 8.3(.16) \\
\text { \% Selected } \\
\text { Typ: } 96(3) \\
\text { Atyp: } 96 \text { (3) }\end{array}$ & $\begin{array}{c}\mathrm{LA}^{-\mathrm{DB}_{1}} \\
\mathrm{~L} \\
\mathrm{D}\end{array}$ & $\mathrm{R}$ & $\begin{array}{l}\text { Rating } \\
\text { Typ: } 4.8(.31) \\
\text { Atyp: } 4.6(.32) \\
\text { \% Selected } \\
\text { Typ: } 7 \text { (3) } \\
\text { Atyp: } 8 \text { (4) }\end{array}$ \\
\hline & & & & $\begin{array}{l}\mathrm{LA}^{2} \mathrm{DB}_{2} \\
\quad \mathrm{~L} \\
\mathrm{D}\end{array}$ & $\mathrm{R}$ & $\begin{array}{l}\text { Rating } \\
\text { Typ: } 5.4(.28) \\
\text { Atyp: } 5.5(.27) \\
\text { \% Selected } \\
\text { Typ: } 61 \text { (3) } \\
\text { Atyp: } 54 \text { (4) }\end{array}$ \\
\hline
\end{tabular}

Figure 2. Display configurations used in Experiments 1 and 2, comprised of distractor placement (absent, in good region, in acceptable region, in bad region) and located object placement (in good region, in acceptable region). The display and condition column provides a label for the configuration, and illustrates the configuration, with $L=$ placement of the located object, $R=$ placement of the reference object, and $D=$ placement of the distractor. The data column provides the mean acceptability rating and standard errors for Experiment 1 and the mean percent and standard error corresponding to selection of the salient object as reference object for Experiment 2. Typ, typical; atyp, atypical.

of the located object in a given region, one can assess whether ratings were influenced by the distractor's placement. The best test of this is within the displays in which the located object is in an acceptable region, comparing across placements of the distractor in the good (LA/DG), acceptable (LA/DA), and bad (LA/DB $\left.1, \mathrm{LA} / \mathrm{DB}_{2}\right)$ regions (see Figure 2). If the relation between the distractor and the reference object is computed, then ratings should be lower, reflecting less acceptability, when the distractor is in a good region (LA/DG), because in this condition, par- ticipants are rating an acceptable above relation between the located object and reference object in the presence of a good above relation between the distractor and reference object (Herskovits, 1986). Further, it is possible that there may be differences when the distractor is in an acceptable (LA/DA) versus a bad $\left(\mathrm{LA} / \mathrm{DB}_{1}, \mathrm{LA} / \mathrm{DB}_{2}\right)$ region. Of particular interest is configuration $\mathrm{LA} / \mathrm{DB}_{1}$, in which the participants rate an acceptable above relation between located object and reference object in the presence of a good above relation between the distractor and located 
object (see Figure 2). A reduction in ratings for this condition would indicate that the distractor's relation with the located object is also computed, with a consequent impact on the interpretation of a spatial relation.

Finally, because Carlson-Radvansky and Tang (2000) found that the typicality of the interaction between the objects influenced the interpretation of spatial relations in some conditions, the displays consisted of trios of objects that included a given reference object and two objects that interacted with this reference object, one typically and one atypically (e.g., a hamburger as reference object, a bottle of mustard that interacts typically; a bottle of pesticide that interacts atypically). Appendix A provides thumbnail sketches of the trios of reference objects and typical and atypical objects. For each display, the located object could be the typical object with the distractor as the atypical object, or the located object could be the atypical object with the distractor as the typical object. An effect due to object typicality would indicate that the functional features of the distractor were also processed. For example, holding constant the display configuration, if ratings were lower when the located object was the atypical object and the distractor was the typical object, this would suggest that participants were assessing the functional interaction among the set of objects, and that this had an impact on the assessment of the spatial relation.

\section{Method}

Participants. Thirty-nine University of Notre Dame undergraduates participated in exchange for partial course credit. All of the participants provided informed consent.

Stimuli. Displays were presented using the e-Prime programming software (Psychology Software Tools, Inc). Eight sets of objects were used; these are shown in Appendix A. The objects consisted of photographs of real objects from Carlson-Radvansky and Tang (2000) and from the Internet, displayed as 24-color bitmaps on a 17 -in. monitor at $640 \times 480$ pixel resolution. Each set contained three objects: a predetermined reference object and two objects that interacted with the reference object, one typically and one atypically. The reference object differed in shape and was the largest object (on average, $3.85 \times 3.98 \mathrm{~mm}$; average area, $15.32 \mathrm{~mm}^{2}$ ), whereas the typical and atypical objects were similar in shape and size [on average, $4.13 \times 2.13 \mathrm{~mm}$; average area, $8.80 \mathrm{~mm}^{2} ; t(7)=2.48$, $p<.05$, one-tailed, for the difference in area between the reference object and the typical and atypical objects]. The configurations that were used are shown in Figure 2. An object in a good above relation was placed at a distance of $68.4 \mathrm{~mm}$ directly above the center of the reference object. An object in an acceptable above relation was positioned above and to the left or right of the reference object at a distance of $94.2 \mathrm{~mm}$ (from center to center). An object in a bad above relation was placed to the left or right of the reference object at a distance of 64.8 or $129.6 \mathrm{~mm}$.

Design. Each participant completed 128 trials, consisting of 32 trials in which the distractor was absent and 96 trials in which the distractor was present. The distractor-absent trials were constructed by crossing the following factors: 2 (typicality of the located object/ distractor) $\times 2$ (placement of located object: good vs. acceptable region) $\times 8$ (stimulus sets). The distractor-present trials were constructed by crossing the following factors: 2 (typicality of target/ distractor $) \times 6$ (display configurations $) \times 8$ (stimulus set). The six display configurations were jointly defined by the placements of the located object and distractor; these are illustrated in Figure 2. Specifically, the located object could appear in a good region, with placement of the distractor in an acceptable region (LG/DA) or in a bad region (LG/DB), or the located object could appear in an ac- ceptable region, with placement of the distractor in a good region (LA/DG), an acceptable region (LA/DA), or a bad region $\left(\mathrm{LA} / \mathrm{DB}_{1}\right.$ and $\mathrm{LA} / \mathrm{DB}_{2}$ ).

Procedure. The sequence of events on each trial was as follows: A fixation point was presented in the center of the screen for $500 \mathrm{msec}$, followed by the sentence The [target] is above the [reference object]. The sentence appeared for $2,000 \mathrm{msec}$, the screen was then blank for $500 \mathrm{msec}$, and then the display was presented along with an acceptability rating scale. The task was to rate the acceptability of the sentence as a description of the picture. The scale was centered at the bottom of the screen within a teal box that segregated it from the scene. The midpoint and endpoints of the scale were numbered and labeled, with 1 corresponding to bad, 5 corresponding to acceptable, and 9 corresponding to good. The display remained present until participants made their response by typing their rating using the side numerical keypad.

\section{Results and Discussion}

Figure 2 provides the displays, the condition labels, and the corresponding mean acceptability ratings and standard errors as a function of the placement of the located object, the placement of the distractor, and the typicality of the located object/distractor. Given the complexity of the results, Table 1 presents the designs and inferential statistics for the critical analyses for Experiment 1; we focus on the theoretically important findings in the text. Analyses subscripted with 1 used participants as the random factor; analyses subscripted with 2 used items as the random factor. $^{2}$ A significance level of $p<.05$ was adopted for all analyses, unless otherwise noted. Critical differences among conditions were computed for follow-up tests for main effects and interactions, based on $95 \%$ confidence intervals constructed using the mean square error term from the relevant main effect or interaction (Loftus \& Masson, 1994); these significant differences are indicated in the text with "> " and "<"; "=" indicates no significant difference between conditions. Partial eta squared $\left(\eta_{\mathrm{p}}^{2}\right)$ is reported as an indication of effect size, describing the proportion of variance that is attributable to a given factor.

Is the presence of the distractor processed? The presence of the distractor was processed, as was reflected in significantly lower ratings for distractor-present displays $(M=6.6)$ than for distractor-absent displays $(M=7.4)$, despite the fact that the spatial descriptions specified which objects (located and reference objects, not distractor) were to be evaluated. ${ }^{3}$ Distractor presence interacted with the placement of the located object, such that the presence of the distractor had a stronger influence when the located object was in the acceptable region [mean difference (present - absent $)=-1.0]$ than when it was in the good region [mean difference (present - absent $)=-.5$ ]. This offers an initial suggestion that the distractor's relation to the reference object was also being computed and compared against the located object's relation to the reference object.

Is the placement of the distractor processed? The processing of the placement of the distractor is indicated if ratings for a given configuration of located and reference objects vary as a function of whether the distractor is in a better, equivalent, or less acceptable relation with the reference object in the display. Two sets of contrasts illustrate that the placement of the distractor was processed. First, holding constant placements of the located object in the 
Table 1

Analysis Design and Inferential Statistics for Experiment 1

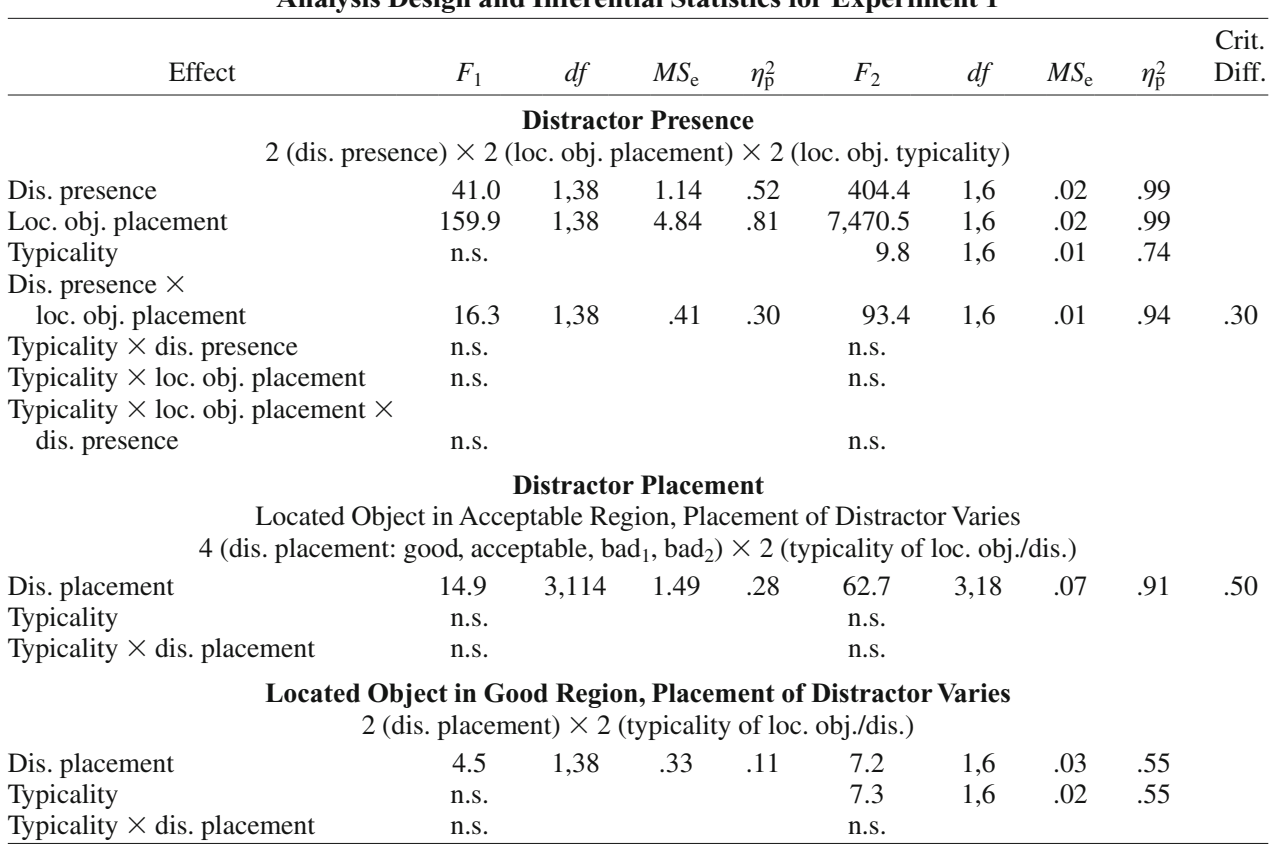

Note-dis., distractor; loc. obj., located object.

acceptable region and comparing across placements of the distractor, the pattern of ratings was $\mathrm{LA} / \mathrm{DB}_{2}(M=5.5)>$ $\mathrm{LA} / \mathrm{DB}_{1}(M=4.7)=\mathrm{LA} / \mathrm{DA}(M=5.1)>\mathrm{LA} / \mathrm{DG}(M=$ 4.2). Second, holding constant placement of the located object in the good region and comparing across placements of the distractor, the pattern of ratings was LG/DB $(M=8.4)>\mathrm{LG} / \mathrm{DA}(M=8.2)$. In both contrasts, as the relation between the distractor and the reference object improved (placements from within a bad region to an acceptable region to a good region), ratings of the relation between the located object and reference object decreased. This pattern suggests that relations between the distractor and the other objects were being computed and compared with the relation with the located and reference objects, following Herskovits (1986). This is counter to the finding by Carlson and Logan (2001) using isolated letters.

Are the properties of the distractor processed? Distractor properties were operationalized as the typical or atypical nature of the interaction between the objects (CarlsonRadvansky \& Tang, 2000; see also Carlson-Radvansky et al., 1999). As reflected in Table 1, there were significant effects of typicality in the items analyses (but not participants analyses) that provide weak support for the idea that the properties of the distractor were processed. Specifically, ratings were lower when the distractor was the more typical object: in the distractor presence analysis (located object atypical/distractor typical $[M=6.9]<$ located object typical/distractor atypical $[M=7.0])$ and in the distractor placement analysis for located object in the good region (located object atypical/distractor typical $[M=8.2]<$ located object typical/distractor atypical $[M=8.4])$. Note, however, that the size of these effects is quite small. In Experiment 2, typicality is again assessed as a distractor property; in Experiment 3, both typicality and the depicted interaction between the objects are examined as additional tests of the processing of the properties of the distractor.

\section{EXPERIMENT 2}

Experiment 2 examined whether the effects of distractor presence, placement, and properties would be observed using a different task. The task used previously (see Carlson \& Logan, 2001; Logan \& Compton, 1996) involved providing the spatial description to participants and asking them to evaluate the spatial relationship between a given located object and a given reference object. This type of task has been referred to as a verification task by Logan and Sadler (1996). In contrast, the predictions made by Herskovits (1986) regarding the suitability of a reference object as a function of the placement of a distractor were in the context of a production task in which the target is given but the reference object may be selected. Logan and Sadler referred to this as a relation judgment task. Importantly, Logan and Sadler argued that the same processes occur within these different routines. Thus, one might also expect to observe effects of the presence, placement, and properties of the distractor within a production task.

In Experiment 2, participants were shown the scenes from Experiment 1, along with a query that identified the located object (e.g., Where is the mustard?), followed by the frame The mustard is The task of the participants was to finish the description, with no restrictions on their completions. The main dependent variable was the frequency with which participants selected the salient object as reference object, as a function of the presence, 
placement, and properties of a nonsalient additional object in the display. By salient object, we mean the object used as the reference object in the descriptions in Experiment 1 (see the list of reference objects in Appendix A); by nonsalient object, we mean the object in the scene that was matched in size and shape to the specified located object. By design, the salient object was perceptually larger and of a different shape than the other objects; it was also functionally salient - able to interact with each of the other two objects within the trio. This designation as salient was verified by an independent group of 18 participants who were shown each trio of objects and asked to pick the object that was perceptually different. Participants selected the salient object significantly more often than chance $[M=.78$, with chance at .33 for three objects; $t(17)=5.14, p<.01]$. This effect was observed in all object sets, as was verified by significant binomial tests of selection of the salient object against chance within each set (all $p \mathrm{~s}<.05$ ).

\section{Method}

Participants. Fifty University of Notre Dame undergraduates participated in exchange for partial course credit. All of the participants provided informed consent. None had participated in Experiment 1.

Stimuli, Design, and Procedure. The scenes from Experiment 1 were used. As in Experiment 1, each participant completed 128 trials: 32 with a located object and the salient object in the displays, and 96 with a located object, salient object, and nonsalient object in the display; these trials were composed of the same factors as in Experiment 1. The sequence of events on a given trial were the same as in Experiment 1, except that the spatial descriptions were replaced with the query Where is the [located object]?, and the display contained the sentence frame The [located object] is Participants were instructed to complete the description by typing on the keyboard. The responses were echoed back to the screen and could be edited as desired. When a participant pressed the Enter key, the description was entered and the next trial began. No restrictions were placed on the length or content of the descriptions.

\section{Results and Discussion}

In total, 6,400 (50 participants $\times 128)$ descriptions were collected. One hundred descriptions were excluded due to experimenter error (see note 2), leaving 6,300 for analysis. We categorized the spatial descriptions into the following types: $\mathrm{E}=$ descriptions that used only environmental features (e.g., The mustard is in the center); $\mathrm{EO}=$ descriptions that used environmental features but also included a reference object (e.g., The mustard is in the upper corner and above the hamburger); $1 \mathrm{~T} 1 \mathrm{O}=$ descriptions that contained one spatial term and one reference object (e.g., The mustard is above the hamburger); $2 \mathrm{~T} 1 \mathrm{O}=$ descriptions that contained two spatial terms and one reference object (e.g., The mustard is above and to the left of the hamburger); $1 \mathrm{~T} 2 \mathrm{O}=$ descriptions that contained one spatial term and two reference objects (e.g., The mustard is above the hamburger and the pesticide); $2 \mathrm{~T} 2 \mathrm{O}=$ descriptions that contained two spatial terms and two reference objects (e.g., The mustard is above the hamburger and to the left of the pesticide) and $2+\mathrm{T} 2 \mathrm{O}=$ descriptions that contained more than two spatial terms and reference objects (e.g., The mustard is above and left of the hamburger and to the right of the pesticide). Table 2 presents the percentages of spatial descriptions by type and display condition, broken down as a function of whether the located object was typical (top panel) or atypical (bottom panel). Note that the overall pattern is very similar across panels (compare summary means), suggesting that the typicality of the located object played a negligible role in participants' selecting the type of spatial description. The most frequent descriptions were environmental, followed by $1 \mathrm{~T} 1 \mathrm{O}$ descriptions.

Evaluating the presence, placement, and properties of the nonsalient object. To evaluate whether the presence, placement, and properties of the nonsalient object were processed, we examined the descriptions containing a reference object in more detail, comparing the percentage of selection of the salient object as reference object across display conditions, following the analyses used in Experiment 1 . When a description included more than one reference object, we coded whether the first-mentioned object was the salient object (see Mainwaring, Tversky, Ohgishi, \& Schiano, 2003, and Taylor \& Tversky, 1996, for according priority to the first-mentioned element in a complex description). These analyses were based on the data for the 25 participants who contributed descriptions containing reference objects in all display conditions; other participants had at least one empty cell, due to exclusive use of environmental descriptions. Mean percentages and standard errors for selection of the salient object as the reference object are provided in Figure 2. Given the complexity of the results, Table 3 presents the designs and statistics for the critical analyses for Experiment 2; we focus on the theoretically important findings in the text.

Is the presence of the nonsalient object processed? When the located object was in the good region, the salient object was selected more often when it was the only object in the display $(M=100 \%)$ than when the nonsalient object was also present $(M=90 \%)(Z=3.0, p=.003) .{ }^{4}$ Similarly, when the located object was in the acceptable region, the salient object was selected more often when it was the only object in the display $(M=100 \%)$ than when the nonsalient object was also present $(M=36 \%)(Z=4.3, p<$ $.001)$. Whereas it may not be surprising (although it was not obligatory) that there was a drop in the selection of the salient object when there was an additional object in the scene, the size of the drop varied as a function of the placement of the located object ( $10 \%$ when the located object was in the good region; $64 \%$ when the located object was in the acceptable region). This is consistent with data from Experiment 1 showing stronger effects of the additional object when the located object was in a less acceptable relation.

Is the placement of the nonsalient object processed? If the placement of the nonsalient object is processed, then the likelihood of selecting the salient object as the reference object should vary as a function of whether the placement of the nonsalient object corresponded to a better, equivalent, or not acceptable relation with the reference object in the display. Two contrasts support this idea (see Table 3). First, holding constant the located object in the acceptable region, and varying the placement of the nonsalient object, the likelihood of selecting the salient object as reference object varied as follows (using the condition labels from Figure 2): $\mathrm{LA} / \mathrm{DB}_{2}(M=58 \%)=\mathrm{LA} / \mathrm{DA}(M=$ $51 \%)>\mathrm{LA} / \mathrm{DG}(M=25 \%)>\mathrm{LA} / \mathrm{DB}_{1}(M=7.5 \%)$. 
Table 2

Percentages and Number of Possible Spatial Descriptions by Type and Display for Experiment 2

\begin{tabular}{|c|c|c|c|c|c|c|c|c|}
\hline & $\mathrm{E}$ & $\mathrm{EO}$ & $1 \mathrm{~T} 1 \mathrm{O}$ & $2 \mathrm{~T} 1 \mathrm{O}$ & $1 \mathrm{~T} 2 \mathrm{O}$ & $2 \mathrm{~T} 2 \mathrm{O}$ & $2+\mathrm{TO}$ & No. Possible \\
\hline \multicolumn{9}{|c|}{ Typical Located Object/Atypical Distractor Object } \\
\hline \multicolumn{9}{|c|}{ Distractor Absent } \\
\hline LG & 26.5 & 16.0 & 57.5 & 0 & 0 & 0 & 0 & 400 \\
\hline LA & 46.5 & 3.5 & 20.7 & 29.3 & 0 & 0 & 0 & 400 \\
\hline \multicolumn{9}{|c|}{ Distractor in Good Region } \\
\hline LA/DG & 37.8 & 9.1 & 24.6 & 2.0 & 2.0 & 13.1 & 11.4 & 350 \\
\hline \multicolumn{9}{|c|}{ Distractor in Acceptable Region } \\
\hline LG/DA & 29.5 & 13.3 & 30.3 & 0 & 0 & 26.9 & 0 & 400 \\
\hline LA/DA & 46.5 & 4.7 & 17.0 & 8.0 & 1.8 & 12.0 & 10.0 & 400 \\
\hline \multicolumn{9}{|c|}{ Distractor in Bad Region } \\
\hline LG/DB & 30.8 & 13.5 & 32.5 & 0.3 & 0.3 & 12.2 & 10.4 & 400 \\
\hline $\mathrm{LA} / \mathrm{DB}_{1}$ & 31.3 & 13.0 & 33.0 & 0.3 & 0 & 12.2 & 10.2 & 400 \\
\hline $\mathrm{LA} / \mathrm{DB}_{2}$ & 48.7 & 1.3 & 3.7 & 3.7 & 1.8 & 33.5 & 7.3 & 400 \\
\hline Mean & 37.2 & 9.3 & 27.4 & 5.5 & 0.7 & 13.7 & 6.2 & \\
\hline Sum & & & & & & & & 3,150 \\
\hline \multicolumn{9}{|c|}{ Atypical Located Object/Typical Distractor Object } \\
\hline \multicolumn{9}{|c|}{ Distractor Absent } \\
\hline LG & 27.8 & 14.7 & 57.5 & 0 & 0 & 0 & 0 & 400 \\
\hline LA & 48.5 & 2.0 & 19.3 & 30.2 & 0 & 0 & 0 & 400 \\
\hline \multicolumn{9}{|c|}{ Distractor in Good Region } \\
\hline LA/DG & 34.5 & 10.8 & 27.8 & 2.0 & 0.7 & 13.2 & 11.0 & 400 \\
\hline \multicolumn{9}{|c|}{ Distractor in Acceptable Region } \\
\hline LG/DA & 30.0 & 14.0 & 29.7 & 0.3 & 0 & 26.0 & 0 & 350 \\
\hline LA/DA & 46.0 & 6.5 & 15.8 & 6.2 & 1.7 & 11.0 & 12.8 & 400 \\
\hline \multicolumn{9}{|c|}{ Distractor in Bad Region } \\
\hline LG/DB & 29.0 & 15.5 & 33.8 & 0 & 0.2 & 12.0 & 9.5 & 400 \\
\hline $\mathrm{LA} / \mathrm{DB}_{1}$ & 30.5 & 14.0 & 32.0 & 0.3 & 0 & 12.5 & 10.7 & 400 \\
\hline $\mathrm{LA} / \mathrm{DB}_{2}$ & 48.3 & 1.0 & 3.3 & 2.7 & 1.0 & 37.0 & 6.7 & 400 \\
\hline Mean & 36.8 & 9.8 & 27.5 & 5.2 & 0.5 & 13.9 & 6.3 & \\
\hline Sum & & & & & & & & 3,150 \\
\hline Overall Mean & 37.0 & 9.5 & 27.5 & 5.3 & 0.6 & 13.8 & 6.3 & \\
\hline Overall Sum & & & & & & & & 6,300 \\
\hline
\end{tabular}

Note-E, description based on environmental features; EO, description with environmental features and a reference object; 1T1O, description with one spatial term and one reference object; $2 \mathrm{~T} 1 \mathrm{O}$, description with two spatial terms and one reference object; 1T2O, description with one spatial term and two reference objects; $2 \mathrm{~T} 2 \mathrm{O}$, description with two spatial terms and two reference objects; $2+\mathrm{TO}$, more than two terms and/or reference objects.

Thus, following Experiment 1, as the relation between the nonsalient object and the reference became more acceptable (DB, DA, DG), the likelihood of selecting the salient object decreased. In addition, there was a big decrease when the nonsalient object formed a good relation with the located object. Indeed, in this condition $\left(\mathrm{LA} / \mathrm{DB}_{1}\right)$, on $82.5 \%$ of the trials, the nonsalient object was selected as the reference object. Second, holding constant the located object in the good region, and varying the placement of the nonsalient object when the relation between the nonsalient object and the reference object became more acceptable, the likelihood of selecting the salient object decreased: Using the condition labels from Figure 2, LA/DB $(M=96 \%)>$ LA/DA $(M=84 \%)$. Taken together, these contrasts indicate that the relations between the distractor and the other objects were being computed and compared with the relation with the located and reference objects, following Herskovits (1986) and consistent with the rating data from Experiment 1.

Are the properties of the distractor processed? Unlike the evidence in the items analyses in Experiment 1, there was no suggestion in Table 3 that the properties of the nonsalient object had an impact on the likelihood that the salient object was selected as the reference object. This issue is further addressed in Experiment 3.

\section{EXPERIMENT 3}

In Experiments 1 and 2, there was consistent evidence that the presence and placement of the distractor were processed, but only a small indication in the items analyses of Experiment 1 that the properties of the distractor were processed. In these experiments, we manipulated object properties by varying the typicality of the interaction with the salient reference object. However, this may be a relatively weak manipulation of object properties. Although there is some support in the literature for the idea that this factor has an impact on the processing of spatial descriptions (Carlson-Radvansky \& Tang, 2000), this effect was only observed in some conditions, and was much smaller than a second manipulationnamely, whether the objects were oriented in a manner that enabled them to interact (e.g., mustard bottle pointing with nozzle down toward the hamburger vs. mustard bottle with nozzle pointing upward away from the hamburger). The interaction manipulation has been observed to influence the interpretation of spatial descriptions in rating (Carlson- 
Table 3

Analysis Design and Inferential Statistics for Nonsalient Object Placement in Experiment 2

\begin{tabular}{|c|c|c|c|c|c|c|c|c|c|}
\hline Effect & $F_{1}$ & $d f$ & $M S_{\mathrm{e}}$ & $\eta_{\mathrm{p}}^{2}$ & $F_{2}$ & $d f$ & $M S_{\mathrm{e}}$ & $\eta_{\mathrm{p}}^{2}$ & $\begin{array}{l}\text { Crit. } \\
\text { Diff. }\end{array}$ \\
\hline $\begin{array}{r}\text { Located Obj } \\
4 \text { (nonsalient object placemer }\end{array}$ & $\begin{array}{l}\text { in Acc } \\
\text { sood, a }\end{array}$ & $\begin{array}{l}\text { Tonsali } \\
\text { table F } \\
\text { ptable }\end{array}$ & $\begin{array}{l}\text { Objec } \\
\text { ion, Pla } \\
\mathrm{d}_{1}, \mathrm{bad}_{2}\end{array}$ & $\begin{array}{l}\text { lacen } \\
\text { nent } \\
<2(t\end{array}$ & $\begin{array}{l}\text { t } \\
\text { Nons } \\
\text { cality }\end{array}$ & nt Ob & $\begin{array}{l}\text { Varies } \\
\text { bject } / 1\end{array}$ & salier & bject) \\
\hline $\begin{array}{l}\text { Nonsalient obj. placement } \\
\text { Typicality } \\
\text { Typicality } \times \\
\text { nonsalient obj. placement }\end{array}$ & $\begin{array}{l}26.0 \\
\text { n.s. }\end{array}$ & 3,72 & 1,050 & .52 & $\begin{array}{l}8.3 \\
\text { n.s. }\end{array}$ & 3,18 & 988 & .58 & $19 \%$ \\
\hline \multicolumn{10}{|c|}{$\begin{array}{l}\text { Located Object in Good Region, Placement of Nonsalient Object Varies } \\
2 \text { (nonsalient object placement) } \times 2 \text { (typicality of located object/nonsalient object) }\end{array}$} \\
\hline $\begin{array}{l}\text { Dis. placement } \\
\text { Typicality } \\
\text { Typicality } \times \\
\text { nonsalient obj. placement }\end{array}$ & $\begin{array}{l}10.3 \\
\text { n.s. }\end{array}$ & 1,24 & 299 & .30 & $\begin{array}{r}20 \\
\text { n.s. }\end{array}$ & 1,6 & 34.9 & .77 & \\
\hline
\end{tabular}

Note-nonsalient obj., nonsalient object.

Radvansky \& Tang, 2000) and free production (CarlsonRadvansky \& Radvansky, 1996) tasks. Moreover, in a task in which participants placed the located object in some spatial relation with respect to the reference object, Carlson and Kenny (2006) showed that whether the objects were able to interact had a significant impact on performance. Accordingly, to further assess whether distractor properties are processed, Experiment 3 employed a rating task similar to that of Experiment 1, with an additional manipulation of whether the objects were oriented in a manner that enabled their interaction. The critical conditions are shown in Figure 3, in which the located object is the mustard, the reference object is the hamburger, and the distractor is the pesticide. There are two independent factors: whether the located object is oriented to interact with the reference object, or whether the distractor is oriented to interact with the reference object.
In addition to these factors, as in the previous experiments, we manipulated whether the located object was typical and the distractor atypical, or vice versa. To keep the number of trials manageable, we included only two display conditions: whether the located object was in a good region or in an acceptable region, crossed with the distractor being present or absent, as shown in Figure 4. Finally, to increase power, we doubled the number of object sets from 8 to 16, with the additional object sets shown in Appendix B.

\section{Method}

Participants. Forty University of Notre Dame undergraduates participated in exchange for partial course credit. All provided informed consent, and none had participated in Experiment 1 or Experiment 2.

Stimuli, Design, and Procedure. The rating task from Experiment 1 was used with 16 stimulus sets, consisting of the 8 sets from Experiments 1 and 2 and 8 additional sets that were constructed

\begin{tabular}{|l|l|l|}
\hline & $\begin{array}{l}\text { Distractor } \\
\text { Interactive }\end{array}$ & $\begin{array}{l}\text { Distractor } \\
\text { Noninteractive }\end{array}$ \\
\hline $\begin{array}{l}\text { Located } \\
\text { object } \\
\text { interactive }\end{array}$ & & \\
\hline $\begin{array}{l}\text { Located } \\
\text { object } \\
\text { noninteractive }\end{array}$ & & \\
\hline
\end{tabular}

Figure 3. Illustration of the located object orientation (interactive vs. noninteractive) and distractor orientation (interactive vs. noninteractive) factors for Experiment 3. 
in a similar manner (see Appendixes A and B). There were 128 distractor-absent trials, made up of the following factors: 2 (located object placement: good vs. acceptable) $\times 2$ (typicality: typical or atypical) $\times 2$ (orientation: interactive vs. noninteractive) $\times 16$ (stimulus sets). There were 256 distractor-present trials, made up of the following factors: 2 (located object/distractor placement: good/acceptable vs. acceptable/good) $\times 2$ (typicality of the located object) $\times$ 2 (orientation of the located object: interactive vs. noninteractive) $\times$ 2 (orientation of the distractor: interactive vs. noninteractive) $\times 16$ stimulus sets. Figure 3 provides examples of the orientation and typi- cality factors, and Figure 4 shows the various placements of the located object as a function of distractor presence. The procedure from Experiment 1 was used.

\section{Results and Discussion}

Figure 4 provides the mean acceptability ratings and standard errors as a function of the placement of the located object, the orientation of the located object and distractor as interactive or noninteractive, and the typicality of the located

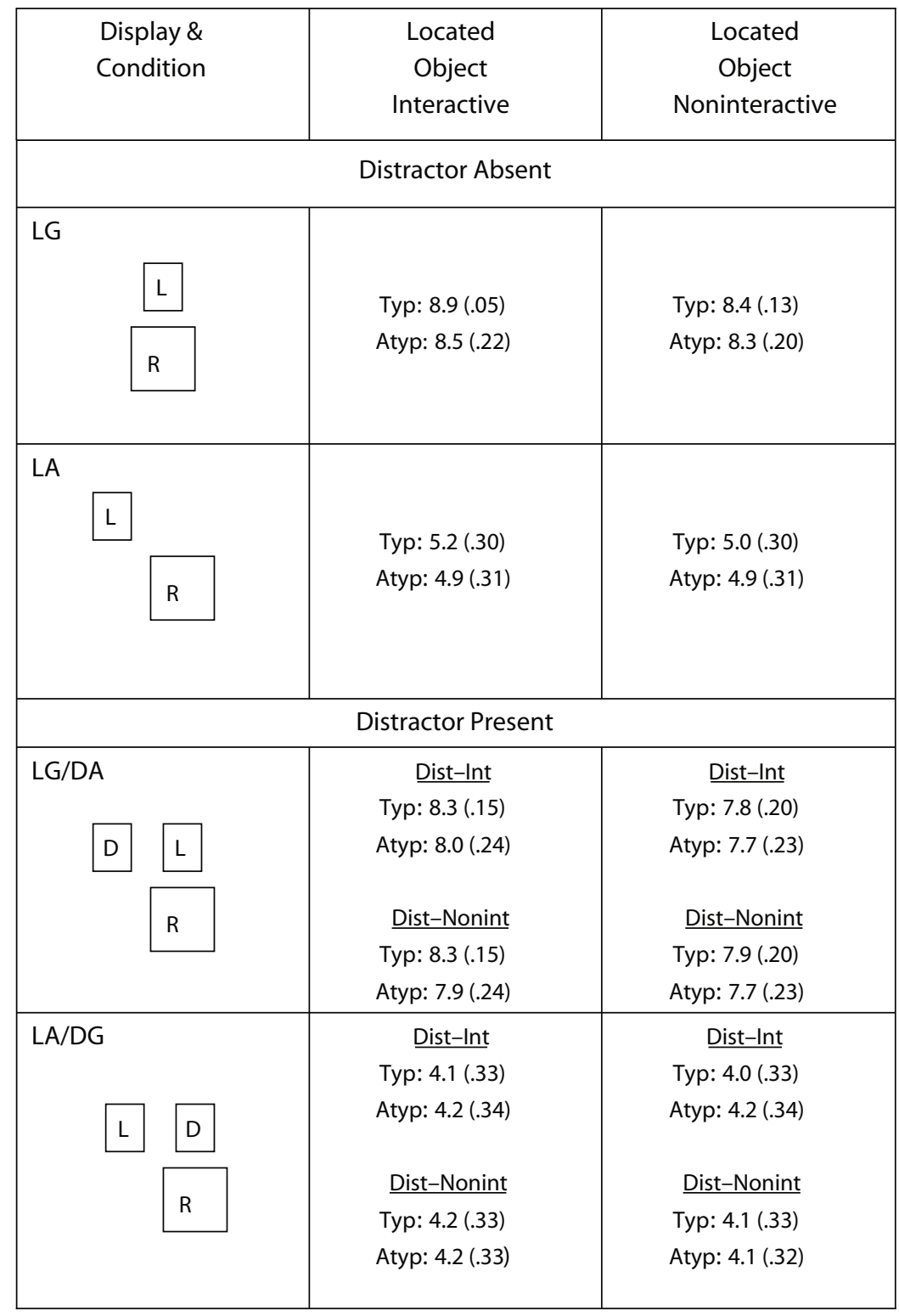

Figure 4. Display configurations used in Experiment 3, comprised of distractor presence versus absence by located object placement in a good or acceptable region. The display and condition column provides a label for the configuration, and illustrates the configuration, with $L=$ placement of the located object, $R=$ placement of the reference object, and $D=$ placement of the distractor. The data column provides the mean acceptability rating and standard errors for Experiment 3. LG, located object in good region; DA, distractor in acceptable region; $\mathrm{DB}$, distractor in bad region; LA, located object in acceptable region; DG, distractor in good region; $\mathrm{DB}_{1}$, distractor in bad region ${ }_{1} ; \mathrm{DB}_{2}$, distractor in bad region ${ }_{2}$; typ, typical; atyp, atypical; dist, distractor; int, interactive; nonint, noninteractive. 
object/distractor. Table 4 presents the designs and inferential statistics for the critical analyses for Experiment 3; we focus on the theoretically important findings in the text.

Is the presence of the distractor processed? The presence of the distractor was processed, as was reflected in significantly lower ratings for distractor-present displays $(M=6.1)$ than for distractor-absent displays $(M=6.8)$. This replicates the results from Experiments 1 and 2. In addition, as an indication that the manipulations of orientation and typicality were effective, ratings for the functionally typical located objects $(M=6.5)$ were significantly higher than ratings for the atypical located objects $(M=6.3)$; ratings for interactive located objects $(M=$ 6.5) were significantly higher than for noninteractive located objects $(M=6.3)$. Moreover, significant two-way interactions among located object placement, located object typicality, and located object orientation (see Table 4) indicate processing of the orientation and typicality of the located object while interpreting the applicability of the spatial term.

With respect to distractor presence, there was a twoway interaction with located object placement in the participants and items analyses, and a three-way interaction among these factors and located object typicality in the items analysis. Specifically, the effect of located object typicality (typical, atypical) was significant for all combinations of located object placement and function (mean differences $>.21$ ) except when the distractor was present and was placed in a good region (mean difference $=$ $-.09)$. In this case, the distractor was the best reference object, and its placement and properties affected the processing of the relation between the other two objects.

Are the placement and the properties of the distractor processed? To assess further whether the distractor placement and properties were processed, we focused on the distractor-present trials, comparing placement, orientation, and typicality of the distractor and located object. Ratings for configurations with the located object in the good region and the distractor in the acceptable region $(M=8.0)$ were significantly higher than for configurations with the located object in the acceptable region and the distractor in the good region $(M=4.1)$. In addition, typical located objects/atypical distractors $(M=6.1)$ were rated significantly higher than atypical located objects/typical distractors $(M=6.0)$. Finally, interactive located objects $(M=6.2)$ were rated significantly higher than noninteractive located objects $(M=5.9)$. This indicates that the properties of the located object were being processed. In contrast, there was no effect of distractor orientation, indicating that, overall, this property of the distractor was not being processed.

Table 4

Inferential Statistics for ANOVAs to Assess Distractor Presence (Top Panel) and Distractor Placement and Properties (Bottom Panel)

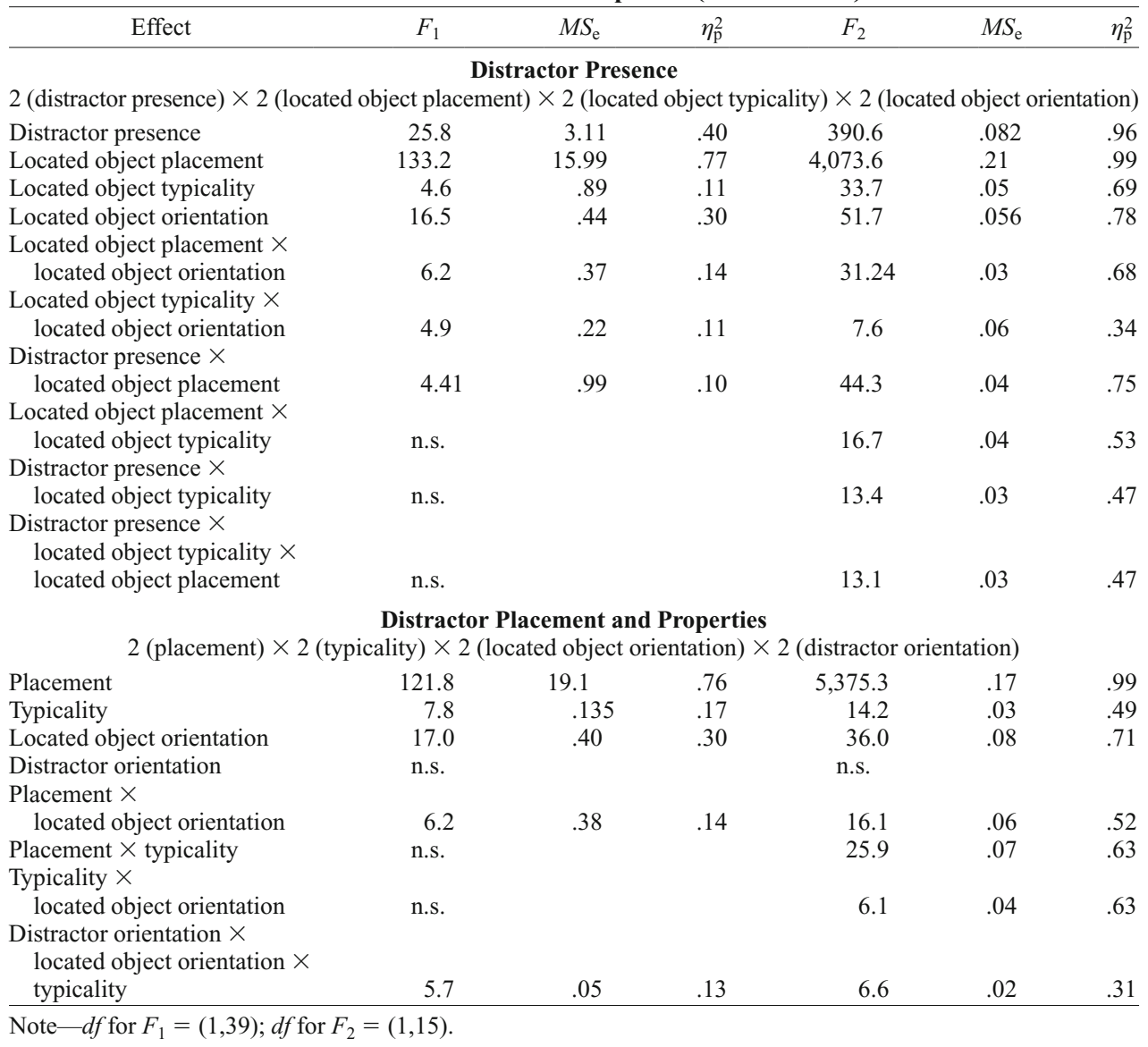


With respect to distractor placement, there was also an interaction between placement and the orientation of the located object, such that there was an effect of orientation when the located object was in the good region (and the distractor was in the acceptable region) [mean difference (interactive - noninteractive) $=.3$ ] but not when it was in the acceptable region (and the distractor was in the good region) (mean difference $=.1$ ). This suggests that when the distractor could serve as a better reference object, the influence of the located object's properties was minimized. With respect to distractor properties, there was a three-way interaction with placement and typicality, such that there was an effect of whether the distractor was positioned to interact only when the distractor was in the good region and was the typical object [mean difference (interactive noninteractive) $=.1]$. In all other conditions (distractor in acceptable region and typical, distractor in acceptable region and atypical, distractor in good region and atypical), there was no impact of distractor orientation (mean differences $<.05$ ). Thus, only when the distractor was both in a preferred placement and was the more typical object was there evidence that its properties were processed. Note also that the effect was quite small.

\section{GENERAL DISCUSSION}

The goal of these experiments was to examine the degree to which the presence, placement, and properties of a distractor are processed during reference frame selection. Using an acceptability rating task, Experiment 1 showed a consistent effect of distractor presence, such that displays with an additional object were rated as less acceptable than displays with only located and reference objects, replicating previous work (Carlson \& Logan, 2001; Logan \& Compton, 1996). There was also a consistent effect of distractor placement, such that ratings were reduced when the distractor and the reference object or the distractor and the located object represented a better example of the spatial relation than did the located object and reference object. This indicates that the relations among all of the objects are being computed. There was limited evidence to support the idea that object properties (in this case, the typicality of the interaction with the reference object) were being processed to a degree that affected the acceptability ratings, despite previous evidence that such characteristics have an impact on the assessment of the relation between the located and reference object (Carlson-Radvansky \& Tang, 2000). This pattern of results was replicated in the rating task of Experiment 3: significant effects of distractor presence and placement, but limited support for the processing of the properties of the distractors, despite doubling the number of object sets and manipulating an additional object feature of the interactiveness of the objects, a factor known to have a strong impact on the interpretation of spatial descriptions (Carlson \& Kenny, 2006; Carlson-Radvansky \& Radvansky, 1996; Carlson-Radvansky \& Tang, 2000). Finally, this pattern was also observed in a production task in Experiment 2, in which the likelihood of selecting the salient object as the reference object was affected by the presence and placement of the distractors but not by their properties.

\section{Letters Versus Objects}

Logan and Compton (1996) and Carlson and Logan (2001) used letters as stimuli, and observed no effect of distractor placement. In contrast, the present study used interacting objects as stimuli, and found an effect of distractor placement but only a weak effect of distractor properties. Why might the identity of the objects but not the letters encourage consideration of the distractors as possible reference objects? Consider the stages proposed by Logan and Sadler (1996) during the apprehension of a spatial description: The located objects and reference objects are spatially indexed and identified, the spatial term is assigned within the display, and the spatial relation between the objects is computed and compared with the assigned spatial term. For both types of stimuli, it seems clear that the distractors (letters or objects) are being considered during spatial indexing and identifying, as reflected in the significant effects of distractor presence. However, it is possible that this identification step has different consequences for letters and objects. For example, given the displays of isolated letters, there is no further processing to do on the letters, once their identity has been checked. In contrast, the identification of the objects may also involve activation of their meaning, including aspects of their function, the way in which they typically interact with other objects, and so forth, consistent with recent links between language comprehension and action and perceptual processes (Barsalou, 1999; Coventry \& Garrod, 2004; Glenberg \& Kaschak, 2002; Zwaan, 2004). This may be especially true given the use of trios of functionally related objects that could interact in the present study. Recent work in action comprehension supports this idea. For example, Bach, Knoblich, Gunter, Friederici, and Prinz (2005) examined the interdependence of functional and spatial information. In one experiment, participants made judgments about the spatial configuration of the objects, and the functional relation between the objects was manipulated to match or mismatch. The spatial decision could be made independently of the functional information, and the key question was whether the functional information would nonetheless have an impact. The finding was that spatial judgments were significantly affected by whether the function matched or mismatched.

However, if such information were fully processed, one might expect to have seen not only an influence of distractor placement, but also a strong effect of distractor properties. Bub, Masson, and Bukach (2003) argued on the basis of imaging data that functional information is not necessarily engaged during all tasks involving objects. For example, object naming tasks are not associated with activation in the premotor cortex, whereas gesture tasks show significant activation. Therefore, it is possible that as participants identify the objects in the present paradigm, the functional information is activated, and encourages computation of the relations among the objects. Reference object selection is then driven in large part by these spatial relations, rather than by the functional properties of the objects. Indeed, Carlson and Kenny (2006) showed that functional information plays a significant role in the interpretation of spatial terms, but only when the interac- 
tion between the objects is enabled; otherwise, judgments are based on geometric information.

An interesting intermediate case between letters and functionally related trios of objects is the use of objects that do not interact but that may occur together. For example, consider again the opening example of spatially describing the location of a cell phone on a desk that has other objects on it, such as a computer monitor and a coffee cup. Using this type of scene, Carlson and Hill (in press) observed that the selection of a reference object was largely based on the spatial relations among the objects, not on perceptual or conceptual features of the objects. This suggests that multiple relations among objects were being computed, consistent with the distractor presence and placement effects observed in the present study. Any potential influence of the properties of the objects would then occur only after the reference object has been selected.

\section{Object Properties: \\ Spatial Location Versus Features}

Previous theoretical work has suggested that the salience of an object is an important criterion for reference object selection, usually defining salience by virtue of perceptual features such as color, shape, size, or mobility (de Vega et al., 2002; Miller \& Johnson-Laird, 1976; Talmy, 1983). Yet the results across the experiments consistently show that it is the placement of the object, rather than its features, that are critical. One way to reconcile these views is to consider a given object's spatial relation to the located object as one of its features. For example, in Experiment 2, there was an increase in the selection of the nonsalient object as reference object when it was placed in a better spatial relation to the located object. These data thus suggest that the most important dimension upon which to select a reference object may be its spatial relation to the located object. Good relations are preferred over acceptable relations, presumably because the former can be expressed quite simply (with single spatial terms), whereas the latter may require a more complex description (combination of terms; e.g., above and to the left of ). This is also reflected in Table 2, in which the number of descriptions with two spatial terms and one reference object (2T1O) was 119 (averaged across typical and atypical objects) when the located object was in the acceptable region and 0 when the located object was in the good region. Moreover, the weak evidence in favor of the processing of the properties of the distractor in Experiment 3 was limited to conditions in which the distractor was in a much better relation to the located object than the reference object. In these cases, it would seem that the distractor was initially selected by virtue of its spatial relation, with its features subsequently processed. That is, its features were processed only because it was initially selected on the basis of its spatial relation.

Notably, the object properties being investigated in the present work are both conceptually driven, based on the function of the objects and their interaction, and perceptually driven, based on properties of size and shape. It is possible that evidence of the processing of object properties might be obtained if other perceptual features were manipulated. Preliminary data (Carlson \& Hill, in press) from a series of experiments in which one object was salient on the basis of its size and color are consistent with the present results in suggesting that spatial location is the critical dimension driving reference object selection. When an object stands in a good relation to the located object, there is a bias to select it as a reference object. The only evidence that the perceptual features of an object were being evaluated was when there were no good relations in the display (e.g., an LA/DA display); however, even in these cases, the effects were not very strong. Thus, spatial features seem to be prioritized over other conceptual and perceptual features during reference object selection, at least for the types of tasks examined in the present article.

\section{Prioritizing Spatial Features}

This prioritization for these spatial features should not be taken as an absolute in at least three senses. First, given that the goal of a spatial description is to locate a given object, an emphasis on spatial features may be most appropriate. However, one might imagine that in tasks in which there is a different goal (e.g., identification or discrimination), different dimensions may be prioritized. Olson's (1970) changing description of the small round white block as a function of its context is a good example of the prioritization of other dimensions on the basis of task or context (see also Brown-Schmidt et al., 2005; Eberhard et al., 1995). Second, spatial features are not strictly independent of other features of an object. For example, Plumert and Hawkins (2001) found a preference for descriptions using in rather than next, due to a bias in favor of containment relations. Containment relations depend integrally on the extent to which an object exerts locational control over a target (for a review, see Coventry \& Garrod, 2004). In addition, there is a strong correlation between the parts and their spatial relations and the functions of an object (see, e.g., Tversky, 2005; Tversky \& Hemenway, 1984). Thus, it may be difficult to separate spatial features from some other perceptual and functional features. Third, additional spatial features - other than the good versus acceptable placements - were examined in the present study. For example, Hund and Plumert (2007) found that relevant distance had a significant impact on the likelihood of judging two objects as nearby. In addition, the presence of an intervening object had a significant effect on the way in which the distances were interpreted. Thus, although the contribution of the present study is to argue that spatial features are important for reference object selection, taken together, these three points suggest that rather than prioritizing one set of features in the absolute, one needs to jointly examine the communicative goals, the interconnection between spatial, conceptual, and perceptual features, and other properties of the display (e.g., the distance between objects, the layout, and the positions of speaker and listener in the scene).

\section{AUTHOR NOTE}

We thank Ashley Haddad, Katie Baron, Katie Osterholz, and Katy Paulus for help in data collection and coding the descriptions in Experiment 2. Correspondence concerning this article should be addressed to L. A. Carlson, Department of Psychology, 118-D Haggar Hall, University of Notre Dame, Notre Dame, IN 46556 (e-mail: lcarlson@nd.edu). 


\section{REFERENCES}

Bach, P., Knoblich, G., Gunter, T. C., Friederici, A. D., \& Prinz, W. (2005). Action comprehension: Deriving spatial and functional relations. Journal of Experimental Psychology: Human Perception \& Performance, 31, 465-479.

Barsalou, L. W. (1999). Perceptual symbol systems. Behavioral \& Brain Sciences, 22, 577-660.

Blocher, A., \& Stopp, E. (1998). Time-dependent generation of minimal sets of spatial descriptions. In P. Olivier \& K.-P. Gapp (Eds.), Representation and processing of spatial relations (pp. 57-72). Mahwah, NJ: Erlbaum.

Brown-Schmidt, S., Byron, D. K., \& Tanenhaus, M. K. (2005). Beyond salience: Interpretation of personal and demonstrative pronouns. Journal of Memory \& Language, 53, 292-313.

Bub, D. N., Masson, M. E. J., \& BuKaCh, C. M. (2003). Gesturing and naming: The use of functional knowledge in object identification. Psychological Science, 14, 467-472.

CARlson, L. A., \& Hill, P. L. (in press). Formulating spatial descriptions across various dialogue contexts. In T. Tenbrink, K. Coventry, \& J. Bateman (Eds.), Spatial language in dialogue. Oxford: Oxford University Press.

Carlson, L. A., \& Kenny, R. (2006). Interpreting spatial terms involves simulating interactions. Psychonomic Bulletin \& Review, 13, 682-688.

CARlson, L. A., \& Logan, G. D. (2001). Using spatial terms to select an object. Memory \& Cognition, 29, 883-892.

CARLSON, L. [A.], \& VAN DER ZEe, E. (2005). Functional features in language and space: Insights from perception, categorization, and development. Oxford: Oxford University Press.

Carlson-Radvansky, L. A., Covey, E. S., \& Lattanzi, K. M. (1999). "What" effects on "where": Functional influences on spatial relations. Psychological Science, 10, 516-521.

Carlson-Radvansky, L. A., \& Radvansky, G. A. (1996). The influence of functional relations on spatial term selection. Psychological Science, 7, 56-60.

Carlson-Radvansky, L. A., \& Tang, Z. (2000). Functional influences on orienting a reference frame. Memory \& Cognition, 28, 812-820.

Clark, H. H. (1973). Space, time, semantics, and the child. In T. E. Moore (Ed.), Cognitive development and the acquisition of language. New York: Academic Press.

Coventry, K. R., \& Garrod, S. C. (2004). Saying, seeing, and acting: The psychological semantics of spatial prepositions. New York: Psychology Press.

Craton, L. G., Elicker, J., Plumert, J. M., \& Pick, H. L., JR. (1990). Children's use of frames of reference in communication of spatial location. Child Development, 61, 1528-1543.

de Vega, M., Rodrigo, M. J., Ato, M., Dehn, D. M., \& Barquero, B. (2002). How nouns and prepositions fit together: An exploration of the semantics of locative sentences. Discourse Processes, 34, 117-143.

Eberhard, K. M., Spivey-Knowlton, M. J., Sedivy, J. C., \& TanenHAUS, M. K. (1995). Eye movements as a window into real-time spoken language. Journal of Psycholinguistic Research, 24, 409-436.

Fillmore, C. J. (1971). Santa Cruz lectures on deixis. Bloomington: Indiana University Linguistics Club.

Glenberg, A. M., \& KaschaK, M. P. (2002). Grounding language in action. Psychonomic Bulletin \& Review, 9, 558-565.

Greenspan, S. L., \& Segal, E. M. (1984). Reference and comprehension: A topic-comment analysis of sentence-picture verification. Cognitive Psychology, 16, 556-606.

HAYWARD, W. G., \& TARR, M. J. (1995). Spatial language and spatial representation. Cognition, 55, 39-84.

HersKovits, A. (1986). Language and spatial cognition: An interdisciplinary study of the prepositions in English. Cambridge: Cambridge University Press.
Hund, A. M., \& Plumert, J. M. (2007). What counts as by? Young children's use of relative distance to judge nearbyness. Developmental Psychology, 43, 121-133.

Loftus, G. R., \& Masson, M. E. J. (1994). Using confidence intervals in within-subject designs. Psychonomic Bulletin \& Review, 1, 476-490.

Logan, G. D., \& Compton, B. J. (1996). Distance and distraction effects in the apprehension of spatial relations. Journal of Experimental Psychology: Human Perception \& Performance, 22, 159-172.

LogAN, G. D., \& SADLER, D. D. (1996). A computational analysis of the apprehension of spatial relations. In P. Bloom, M. A. Peterson, L. Nadel, \& M. Garrett (Eds.), Language and space (pp. 493-529). Cambridge, MA: MIT Press.

Mainwaring, S. D., Tversky, B., Ohgishi, M., \& Schiano, D. J. (2003). Descriptions of simple spatial scenes in English and Japanese. Spatial Cognition \& Computation, 3, 3-42.

Miller, G. A., \& Johnson-Laird, P. N. (1976). Language and perception. Cambridge, MA: Harvard University Press.

Olson, D. (1970). Language and thought: Aspects of a cognitive theory of semantics. Psychological Review, 77, 143-184.

Plumert, J. M., Carswell, C., De Vet, K., \& Ihrig, D. (1995). The content and organization of communication about object locations. Journal of Memory \& Language, 34, 477-498.

Plumert, J. M., Ewert, K., \& Spear, S. J. (1995). The early development of children's communication about nested spatial relations. Child Development, 66, 959-969.

Plumert, J. M., \& Hawkins, A. M. (2001). Biases in young children's communication about spatial relations: Containment versus proximity. Child Development, 72, 22-36.

Talmy, L. (1983). How language structures space. In H. L. Pick \& L. P. Acredolo (Eds.), Spatial orientation: Theory, research, and application (pp. 225-282). New York: Plenum.

TAYlor, H. A., \& TVERsky, B. (1996). Perspective in spatial descriptions. Journal of Memory \& Language, 35, 371-391.

TVERsKy, B. (2005). Form and function. In L. [A.] Carlson \& E. van der Zee (Eds.), Functional features in language and space: Insights from perception, categorization and development (pp. 332-347). Oxford: Oxford University Press.

Tversky, B., \& Hemenway, K. (1984). Objects, parts, and categories. Journal of Experimental Psychology: General, 113, 169-193.

ZWAAN, R. A. (2004). The immersed experiencer: Toward an embodied theory of language comprehension. In B. H. Ross (Ed.), The psychology of learning and motivation: Advances in research and theory (Vol. 44, pp. 35-62). San Diego: Academic Press.

\section{NOTES}

1. Carlson and Logan (2001) presented their data collapsing across configurations $\mathrm{LA} / \mathrm{DB}_{1}$ and $\mathrm{LA} / \mathrm{DB}_{2}$.

2. Due to experimenter error, the paint can stimulus was improperly presented in the LG/DA and LA/DG conditions. Consequently, subject means for these conditions are based on seven rather than eight observations, and this set is excluded from the item analyses.

3. As shown in Table 1, this finding came from a 2 (distractor presence) $\times 2$ (located object placement) $\times 2$ (located object typicality) repeated measures ANOVA. As shown in Figure 2, different numbers of conditions fall into the cells of this design, as follows: Each distractorabsent cell had one condition for each placement of the located object, the distractor-present cell with the located object placed in the good region had two conditions, and the distractor-present cell with the located object placed in the acceptable region had four conditions. Prior to analysis, the conditions within each cell were averaged; therefore, the levels of the factors in the anova were equally weighted.

4. Nonparametric Wilcoxon tests were used here because at least one of the conditions had a percentage of $100 \%$ (and hence, no variability). 
APPENDIX A

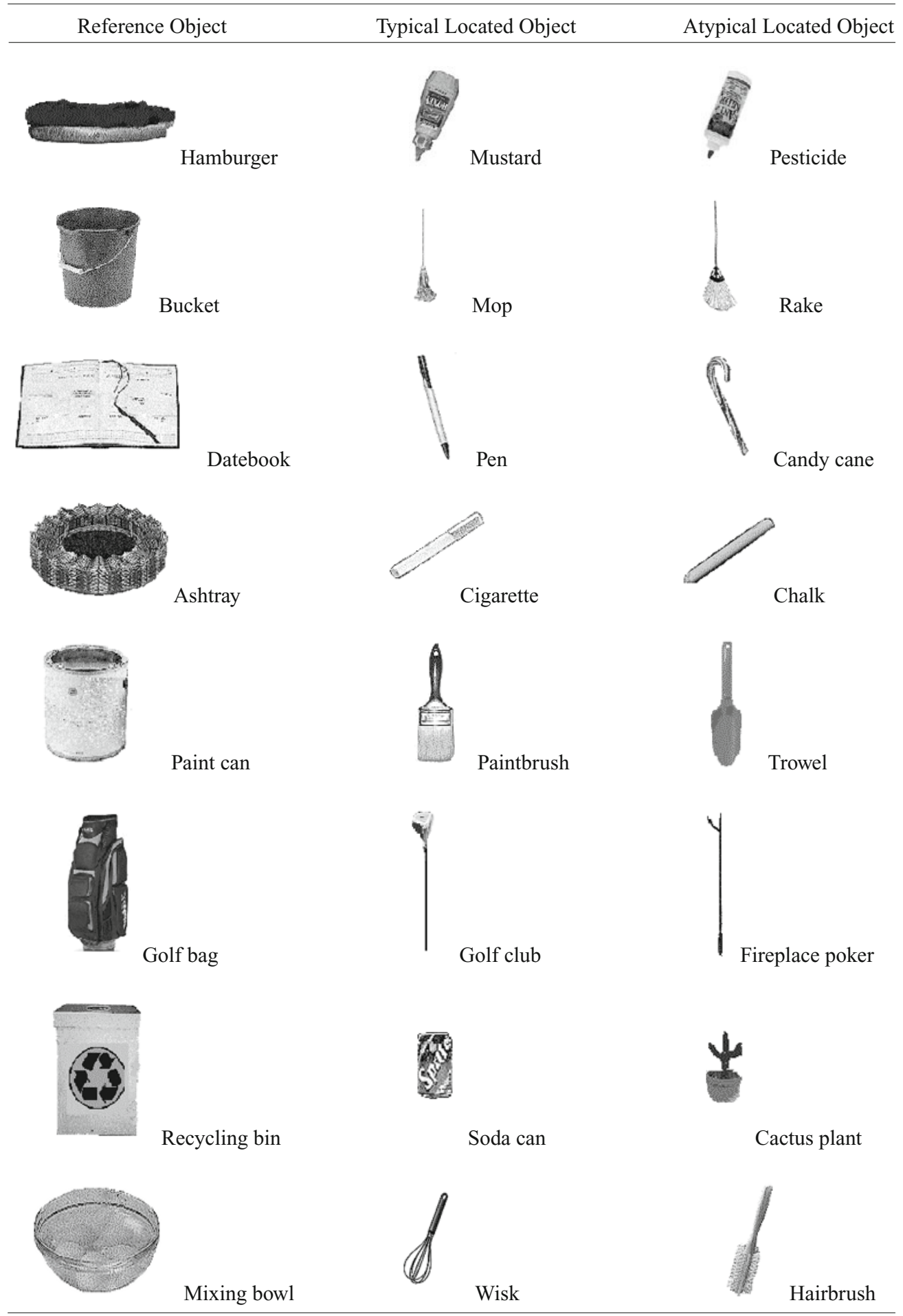


APPENDIX B

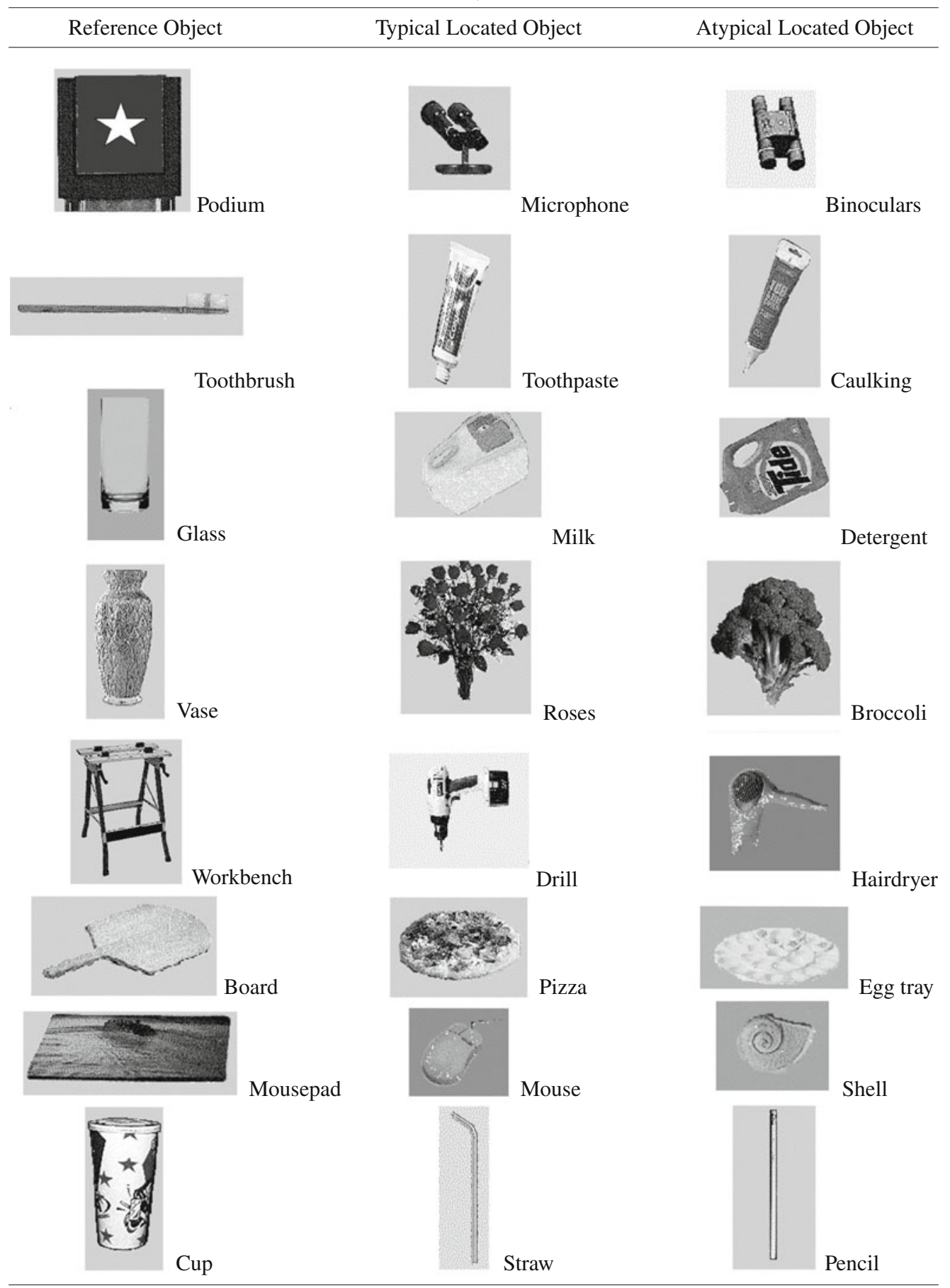

(Manuscript received March 4, 2007;

revision accepted for publication August 25, 2007.) 